\title{
La importancia de la investigación del lugar de la muerte y de la entrevista familiar en casos de muerte súbita e inesperada del
} lactante. The importance of the death scene investigation and family interview in cases of sudden unexpected infant death.

B. Aguilera ${ }^{1}$ y M.P. Suarez-Mier ${ }^{1}$

\begin{abstract}
RESUMEN
El Síndrome de Muerte Súbita del Lactante (SMSL) es la principal causa de muerte súbita e inesperada del lactante durante el período postneonatal en los países desarrollados. Se define como la muerte súbita e inesperada de un lactante que permanece inexplicada después de la revisión de la historia clínica, examen del lugar de la muerte y realización de una autopsia completa, incluyendo análisis complementarios. En los últimos tiempos se ha puesto en evidencia que casos de muerte intencional fueron catalogados como SMSL por una deficiente investigación de las circunstancias y el lugar de la muerte. Queremos hacer hincapié en la importancia de esta parte de la investigación postmortem y resumimos algunas de las recomendaciones publicadas al respecto..
\end{abstract}

Palabras clave: Muerte Súbita del Lactante, investigación del lugar de la muerte.

\section{ABSTRACT}

The sudden infant death syndrome (SIDS) is the principal cause of sudden unexpected death during the post neonatal period in developed countries. It is defined as the sudden death of an infant under one year of age which remains unexplained after a thorough case investigation including a complete autopsy with the relevant analyses, examination of the death scene and a review of the clinical history. Recently it was shown that cases of infanticide had been diagnosed as SIDS because of poor investigation of the circumstances and scene of death. In this report we stress the importance of this part of the post mortem investigation and we summarize some of the guidelines published on the subject.

Key words: Sudden Infant Death, death scene investigation.

Correspondencia: Dra. Beatriz Aguilera.Sección de Histopatología. Instituto Nacional de Toxicología. C/ Luis Cabrera, n 9. 28002 Madrid. Teléfono: 91-562 84 69. Fax: 91-563 69 24. E mail: b.aguilera@mju.es

\footnotetext{
${ }^{1}$ Especialista en Anatomía Patológica. Sección de Histopatología. Instituto Nacional de Toxicología. Madrid.
} 
El Síndrome de Muerte Súbita del Lactante (SMSL) es la primera causa de muerte inesperada durante el primer año de vida en países occidentales, si se excluye el periodo neonatal. Fue definido por Beckwith en 1969, como la muerte de un lactante o niño pequeño que es inesperada por la historia y en donde el examen postmortem no encuentra una adecuada causa de muerte $[\mathrm{I}]$.

El estudio comparativo de casos de muerte súbita e inexplicada y de casos de muerte súbita por algunas enfermedades naturales y por causas violentas demostró que una autopsia completa no era suficiente para el diagnóstico de un SMSL. Por ello en 1989, el National Institute of Child Health and Human Development, incluyó la necesidad del examen del lugar de la muerte y la revisión de la historia clínica para establecer el diagnóstico [2].

La autopsia debe ser completa y en todos los casos, además de un muestreo completo para estudio histopatológico (encéfalo, médula espinal, músculo y bloque cérvico-tóracoabdominal), debe incluir muestras para estudio microbiológico, análisis químico-toxicológico, determinación de glucosa y electrolitos en humor vítreo e investigación de toxina botulínica en heces [3].

En la última década, desde que se han hecho campañas recomendando que los recién nacidos duerman en posición supina, en los países occidentales se ha observado una considerable reducción de casos de SMSL [4]. Por otra parte, desde la introducción de la investigación de las circunstancias y del lugar de la muerte, así como de los antecedentes familiares, se ha comprobado que muchos casos catalogados como SMSL correspondían en realidad a muertes accidentales e intencionadas.

La muerte de un lactante solo se puede catalogar de SMSL si se cumplen los siguientes requisitos:

- Se ha efectuado una autopsia completa y los hallazgos son los compatibles con el SMSL (edema pulmonar, petequias en órganos intratorácicos).

- No hay evidencias macro-microscópicas de trauma, quemaduras, de un proceso patológico o error congénito del metabolismo que pueda explicar la muerte.

- No hay evidencias de trauma al examen radiológico del esqueleto.

- No hay evidencias de exposición a alcohol, drogas, monóxido de carbono u otros tóxicos.

- El examen del lugar de la muerte y la revisión del historial clínico (que incluya antecedentes de la gestación y parto) son negativos [5].

Los hallazgos autópsicos en casos de SMSL pueden ser indistinguibles con los casos de sofocación, ya sea accidental o intencional, si la presión sobre la cara se ha hecho con un objeto blando o con la mano [6]. Por ello es fundamental que el forense investigue el lugar de la muerte e interrogue a los familiares más próximos o a la persona que estaba al cuidado del lactante en las últimas 24 horas previas al fallecimiento. El medir la temperatura ambiente o verificar la ropa de abrigo y de cama que tenía en el momento del fallecimiento, permite sospechar una hiper o hipotermia como posible mecanismo de muerte [7].

El Centro para el Control de Enfermedades de Estados Unidos publicó unas recomendaciones para la investigación del lugar de la muerte en casos de muerte súbita e inesperada del lactante que resumimos a continuación [8]: 
- Se debe investigar el sitio en que el lactante se encontró sin respuesta o muerto, así como el sitio en que lo atendieron y entrevistar a todas las personas relacionadas. Por ejemplo, si lo encuentran sin respuesta o sin vida en la guardería y el personal lo traslada a un ambulatorio, hay que revisar el domicilio, la guardería e interrogar a quienes le atendieron en el ambulatorio o iniciaron las maniobras de reanimación.

- Recoger toda la información que debe incluir el historial médico y las circunstancias de la muerte.

- Recomiendan llevar una muñeca de tamaño natural para reproducir en la forma más veraz como ocurrieron los hechos: sitio, posición, descripción de las ropas, tipo de colchón, objetos que había en la cuna. Indagar si compartía cama o sofá en el momento del incidente.

- Si el lactante todavía está presente al realizar el examen del lugar de la muerte, documentar la distribución de las livideces, determinar presencia de rigidez y la posición del cuerpo (descartar sofocación por ropa de cama, por deslizamiento de la cabeza entre la cuna y el colchón o por lazos de la ropa del bebé).

- Ver el estado de conservación y limpieza de la habitación y del hogar. Buscar alcohol, drogas de abuso, fármacos u otras substancias potencialmente tóxicas en la casa.

- Buscar y preguntar sobre el uso de remedios caseros o populares como hierbas, agua de anís, etc.

- Verificar si existe agua potable o la fuente de dónde se obtiene.

- Tomar la temperatura del lactante y de la habitación en que estaba en el momento del deceso.

- Comprobar o averiguar el tipo de calefacción usado, la distancia que existe con la cuna o si existe una fuente que pueda desprender monóxido de carbono.

- Determinar la cantidad y calidad de la ropa de cama que había en la cuna y si estaban húmedas por sudoración.

- Recoger el pañal y la ropa que usaba en el momento del incidente.

- Recoger muestras del último biberón y de la fórmula que usaba.

- Verificar si había cables o electrodomésticos vecinos a la cuna o donde fue hallado el lactante. Determinar su estado.

- Revisar si la bañera o el lavabo estaban llenos de agua, ver si hay ropas mojadas del lactante en el baño y dejar constancia si el cabello del lactante estaba mojado, húmedo o seco.

- Inspeccionar el o los servicios buscando restos de sangre.

- Buscar en la basura o por la habitación bolsas de plástico que pudieran haber servido para asfixiar al niño (Se deben buscar restos de saliva).

- Determinar si cerca de la cuna había algún cordón como de una cortina que pudiera en forma accidental haber estrangulado al bebé.

- Verificar si el colchón ajusta bien a la cuna, si no, medir la distancia que queda entre el colchón y la cuna.

- Documentar el estado de la cuna y de todos los objetos que en ella había. Recoger todos los objetos que sean tan pequeños que potencialmente pudieran haber obstruido la vía aérea del lactante.

- Determinar si el lugar donde fue encontrado muerto el lactante era el sitio donde dormía habitualmente (cuna, cama de los padres). 
- Si compartía cama, relatar con quién y determinar el sitio preciso que cada uno ocupaba en el momento del incidente (Ejemplo: estaba entre los dos padres, o estaba en la cama matrimonial al lado de la madre).

- Hacer inventario de las medicinas que había en el hogar y de quien las usaba.

- Verificar la existencia de plantas y tipo que hay en el domicilio.

- Determinar si el hogar estaba infectado por insectos, roedores, o la existencia de animales de compañía.

- Ver si existe en el hogar olor que indique el uso reciente de productos de limpieza, pinturas o quita pinturas que puedan desprender gases tóxicos.

- Si el niño usaba monitor, este debe recogerse y precintarse, con el fin de analizar su registro y las incidencias ocurridas.

- Tomar fotografías o hacer esquemas de los hallazgos.

Gracias a la investigación del lugar y circunstancias de la muerte, Bass y cols. demostraron que en 18 de 26 casos de posibles SMSL, las causas de muerte fueron otras: asfixia accidental, sofocación por compartir la cama con un adulto o hipertermia [9].

Por otra parte, en una serie de 289 casos estudiados en el Instituto de Medicina Forense de Oslo entre 1984 y 1998, tras la investigación del lugar y reconstrucción de lo sucedido demostraron 3 muertes accidentales, tres casos de negligencia y un caso de asesinato [10].

El grupo australiano de Byard y cols. encontraron la causa tras el examen del lugar de la muerte en un $3 \%$ de los casos [ I I]

Junto a la investigación del lugar de la muerte es fundamental averiguar:

- Si la policía ha sido llamada antes a ese domicilio, cuántas veces y por qué causa.

- Si esa familia ha requerido de asistencia social y si es así, entrevistar a los asistentes sociales.

- Determinar si hay historia documentada de maltrato a los niños en la familia.

- Indagar si hay hermanos, su salud y sobre el antecedente de fallecimiento de otros hermanos.

- Indagar sobre cambios de domicilio y en lo posible determinar dirección de estos últimos [8].

Si bien es cierto que es imposible diferenciar en la autopsia un caso de SMSL de una sofocación hecha con un objeto blando, el Committee on Child Abuse and Neglect de la Academia Americana de Pediatría estima que ciertas circunstancias indican la posibilidad de sofocación intencional:

- Antecedente de crisis recidivantes de cianosis, apnea o episodios aparentemente letales (ALTE) bajo el cuidado de una misma persona.

- Lactante mayor de 6 meses en el momento del fallecimiento.

- Antecedente de muerte inesperada e inexplicada en uno o más hermanos.

- La muerte simultánea o casi simultánea de dos mellizos o gemelos.

- El antecedente de fallecimientos previos de otros niños bajo el cuidado de la misma persona.

- El hallazgo de sangre en la nariz o boca en asociación con un episodio aparentemente letal (ALTE) [5]. 
La posibilidad que un lactante fallezca en forma súbita e inesperada se ha estimado que es de I/I 000 nacidos vivos. Pero la posibilidad que esto ocurra por dos veces en la misma familia, es de $1 / 1000.000$. Por lo tanto, es muy improbable que dos o más niños de una misma familia fallezcan de causas naturales [12]. Por ello es fundamental averiguar sobre el antecedente de fallecimientos infantiles previos en esa familia.

Sin embargo, pueden ocurrir muertes naturales múltiples cuando existen causas hereditarias como son los síndromes que ocasionan arritmias letales, tales como el síndrome del QT largo [13,14] o el síndrome de Brugada [15] y errores innatos del metabolismo. También puede ocurrir cuando persiste en la familia el mismo tipo de cuidado a los lactantes y que pueden ser de riesgo para su vida (si no se ha corregido el dejarlos en decúbito prono, sobre abrigados, con las mantas cubriendo la cabeza o en un ambiente muy caliente).

Si todo esto se ha investigado, el antecedente de dos muertes inesperadas de un lactante en la misma familia obliga a una meticulosa investigación por un equipo multidisciplinario que incluya a pediatras, forenses, patólogos y personal policial, ojalá especializado en estos casos, ya que lo más probable es que el fallecimiento no sea por causas naturales.

Joaquín Lucena como miembro fundador del Comité Ejecutivo del Grupo de Trabajo para el Estudio y Prevención de la Muerte Súbita Infantil (GEPMSI) de la Asociación Española de Pediatría (AEP) y responsable de los aspectos médico legales del grupo, publicó un protocolo de estudio multidisciplinario que incluía aspectos relacionados con la historia clínica y el examen del lugar de la muerte $[16,17]$.

En nuestra experiencia, con cerca de trescientos casos de muerte súbita del lactante estudiados en la última década en el INT de Madrid, el protocolo casi nunca se rellena, la investigación del lugar de la muerte por el forense pocas veces se realiza y, debido a la dispersión geográfica de los casos, la valoración por un equipo multidisciplinario de cada caso, es excepcional.

Es posible que esta sea la razón de que en una serie de 173 casos estudiados entre 1995 y $200 \mathrm{I}$, tan solo tengamos constancia de 2 muertes por sofocación accidental, identificadas gracias a los datos obtenidos por los médicos forenses [18].

\section{CONCLUSIÓN:}

El diagnóstico de Síndrome de Muerte Súbita del Lactante es un diagnóstico de exclusión y requiere de un estudio multidisciplinario, preferiblemente por personal entrenado en este campo específico, que no deben olvidar que aquellas familias que sufren el fallecimiento inesperado de un lactante, están pasando probablemente por el momento más traumático de sus vidas y que los estudio deben hacerse teniendo en cuenta el gran dolor de los afectados. Una vez obtenidos los resultados, los familiares deben recibir una explicación sobre cual ha sido la causa de la muerte inesperada del lactante, si se le encuentra, y sino, estos padres que por lo general están angustiados y con sentimientos de culpabilidad, deben recibir explicaciones comprensibles sobre el SMSL.

Por otra parte, las circunstancias del fallecimiento, el historial clínico, la reacción de la familia, el obtener datos contradictorios sobre las circunstancias de la muerte, un embarazo no controlado, nivel socioeconómico bajo, etc, nos pueden poner sobre la pista que la muerte del lactante no es natural sino intencional. 


\section{BIBLIOGRAFÍA:}

I.- Beckwick JB. Discussion of terminology and definition of the sudden infant death syndrome. Proceedings of the Second International Conference on the Causes of sudden death in infants. University of Washington Press, Seattle, 1970. Citado por Rognum T0 en Definition and pathologic features. En: Sudden Infant Death Syndrome editado por Byard y Krous, Arnold, London 200I, cap. 2: 430.

2.- Willinger M, James S, Catz C. Defining the SIDS: Deliberations of an expert panel convened by The National Institute of Child Health and Human Development. Pediatr Pathol 1991;|I:677-684.

3.- Normas de Remisión de Muestras para el Estudio del Síndrome de la Muerte Súbita del Lactante del Instituto Nacional de Toxicología. Ministerio de Justicia, septiembre de 1993.

4.- Hauck Fern R. Changing Epidemiology. En: Sudden Infant Death Syndrome editado por R. Wyard y H. Krous, Arnold, London, 200I, cap. 3: 31-57.

5.- Committee on Child Abuse and Neglect of American Academy of Pediatrics: Distinguishing Sudden Infant Death Syndrome from Child Abuse Fatalities. Pediatrics 2001; 107:437-44I.

6.- Valdés-Dapena M, McFeeley P, Hoffman HJ et al. Histopathology Atlas for the Sudden Infant Death Syndrome. Armed Forces Institute of Pathology, Washington, 1993 pp II8 y 144.

7.- Byard RW and Krous HF. Differential diagnosis of sudden infant death. En: Sudden Infant Death Syndrome. Problems, Progress and Possibilities. Editado por R. Byard and HF. Krous, Arnold 200I, London, Cap 12:214.

8.- Hanzlick R. Death Scene Investigation. En: Sudden Infant Death Syndrome. Editado por R. Byard y H. Krous, Arnold 200I, London, Cap 4:58-65.

9.- Bass M, Kravath RE, Glass L. Death-scene investigation in sudden infant death. N Engl J Med 1986;315:100-105.
10.- Rognum T.O. Definition and Pathological features. En: Sudden Infant Death Syndrome. Problems, Progress and Possibilities. Editado por Byard y Krous. Arnold, London 200I, cap 2:4-30.

II.- Mitchell E, Krous HF, Donald T, Byard RW. An analysis of the usefulness of specific stages in the pathologic investigation of sudden infant death. Am J Forensic Med Pathol 2000;21:395-400.

12.- Meadow R. Unnatural sudden infant death. Arch Dis Child 1999;80:7-14.

13.- Schwartz PJ, Stramba-Badiale M, Segantine A, et al. Prolongation of the $\mathrm{QT}$ interval and the sudden infant death syndrome. N Engl J Med 1998;338:1709-14.

14.- Schwartz PJ, Priori SG, Dumaine R, et al. A molecular link between the sudden infant death syndrome and the long $Q T$ syndrome. N Engl J Med 2000;343:262-7.

I5.- Napolitano C, Memmi M, Giordano G, et al. Clinical and Molecular evidences supporting the role of Brugada Syndrome as a determinant of sudden infant death. European Heart J 2000;21:449 (abstract).

16.- Lucena J. Importancia de la aportación Médico-Forense en la muerte súbita del lactante: presentación de un estudio multidisciplinar epidemiológico, clínico, anatomo-patológico y médico-legal. Rev. Esp. Med. Legal I99I; Año XVIII:73-85.

17.- Lucena Romero J. Protocolo de estudio multicisciplinario: epidemiológico, clínico, patológico y médico-legal en casos de muerte súbita infantil en Síndrome de la Muerte Súbita del Lactante (SMSL) Libro Blanco. Grupo de Trabajo para el Estudio y Prevención de la Muerte Súbita Infantil de la Asociación Española de Pediatría (GEMPSI de la AEP) Coordinador Camarasa Piquer F. Ergón, 1996, Madrid. 18.- Aguilera B. y Suarez-Mier MP. Postmortem investigation of sudden infant death in Spain (1995-200I) Poster. En: Conference Handbook on The Seventh SIDS International Conference, Florence, August 3I-september 4, 2002: pp I28. 